\title{
Economic Burden of Surgical Site Infections in Cardiac Surgery
}

\section{Pascal M Dohmen*}

Department of Cardiac Surgery, Heart Centre Leipzig, University of Leipzig, Sanoxy, Germany

Keywords: Surgical site infections; Mediastinitis; Economic burden

\section{Introduction}

Superficial and deep post-sternotomy infections after cardiac surgery remains an important cause of morbidity and mortality, described in the literature with an incidence of up to $15 \%$. The normal skin flora is a complex ecosystem formed by the normal microflora of $10^{14}$ microbes and $10^{13}$ mammalian cells [1]. The intact skin is resistant again microbial invasion due to relative dryness, cell-mediated immunity and antibody production. However, the type and virulence as well as the quantity of inoculated bacteria are on the other hand important factors to implicate a Surgical Site Infection (SSI).

Sharma et al. [2] showed in a study on SSI in patients following coronary artery bypass grafting, that Staphyloccocus aureus, which is part of the transient skin flora is twice as often seen in deep SSI compared with superficial post-sternotomy infections, 30/51 (58.8\%) versus 16/71 (22.5\%) respectively. Coagulase-negative staphylococci, which is part of the residential skin flora, are more present in superficial wound infections compared with deep SSI, 23/71 (32.4\%) versus 4/51 (7.8\%) respectively. This study furthermore showed, as the virulence of the coagulase-negative staphylococci is not as severe as $S$. aureus, the cause of surgical wound cultures between superficial and deep SSI was similar high for coagulase-negative Staphylococci. Adequate disinfectant to reduce the quantity of potential inoculating microbes of the transient and/or residential skin flora is of enormous importance and should not be underestimated.

Mediastinitis, the most serious form of deep SSI, show an actuarial survival of $84.6 \pm 7.1 \%$ if a Methicillin-Susceptible S. aureus (MSSA) is cause of SSI, however survival statistically significant decreases to $60.0 \pm$ $12.6 \%$ at one month follow-up in case of Methicillin-Resistant S. aureus (MRSA) post-sternotomy mediastinitis $(\mathrm{p}=0.037)$ [3]. Interestingly this study of Mekontso-Dessap et al. [3] showed in a highly selected patient population a three year survival of only $26.3 \%$ in MRSA poststernotomy mediastinitis versus $79.0 \%$ in MSSA post-sternotomy mediastinitis.

On the other hand there is an economic burden associated with SSI, especially in patients suffering from post-sternotomy mediastinitis after cardiac surgery. The literature normally reports only on hospital costs due to prolonged hospitalization and re-admission. Generally the prolonged hospital stay of patients suffering from SSI is 10 days, in cardiac surgery even 12 days, which results in extra costs for the European health service of approximately 19 billion Euros each year [4]. Cost of additional hospitalization days due to SSI are not only dependent on hospital facility care (primary, secondary or tertiary), but also on particularly country within the European Union, presented in the study of Leaper et al. [5] with additional hospitalization costs calculated as low as 170 Euro/day in Spain with up to 413 Euro/day in Italy. The hospital cost due to additional hospitalization days to treat SSI is only a limited part apart of the direct health costs of patients treated for SSI. The total direct health costs are a summary of the costs due to prolonged hospital stay, antibiotic costs, other hospital costs and primary health costs. The prolonged hospital stay costs are generally direct hospital costs which can be divided in fixed costs (Building, utilities, equipment, environment, etc.). However, additionally there are variable costs such as medication, consultation, treatment, surgery, devices such as VAC therapy, examination, drug associated costs by monitoring adjunctive therapy and adverse events. This part is variable, however generally as high as the fixed costs. Antibiotic therapy is always considered, especially in case of the use of high sophisticated reserve antibiotics, as an important part of variable costs. In reality this part is only as high as $7 \%$ and therefore not the limited part of the costs. Furthermore sufficient early antibiotic therapy will reduce the hospital stay by avoiding complications, which has a greater impact on the costs.

The most important part of the economic burden due to SSI are the indirect costs due to temporary or permanent incapacity to work, expressed in years of productive life lost, morbidity, mortality, income lost by family members, forgone leisure time, travel costs, home care costs. Limited data are only available in the literature since it is generally difficult to calculate. These costs however have an enormous impact of the total costs associated with SSI. Alfonso et al. [6] performed a study in Spain with the provocation title "are we really seeing the total costs of surgical site infections?". This study showed interesting data of patients with major surgery in Spain, included 103 patients undergoing cardiac surgery.

Health costs are higher in the patients over the age of 60 years, with the highest rate in the patient population between the age of 71 and 80 years $(377,594 \$$ total health cost) compared with the group of patients below the age of 40 years with only $51,490 \$$. Additionally there are caregiver costs, generally provided by family, neighbours or friends in helping the patient with different basic and instrumental activities. These costs were again the highest in the older patients, especially in the group between 71 and 80 years of age, respectively $717,381 \$$, which was equal to $65 \%$ of the total costs.

On the other hand there are the indirect costs which were higher in the younger patients under the age of 40 years, respectively 2,418,308\$ due to years of productive life lost. Also important in this study was that the number of patients below the age of 40 years were $8 / 106(7.5 \%)$ with SSI. The total number of patients above the age of 60 years was $74 / 106$ (69.8\%). These analyzes shows that the total costs, means direct and indirect costs, in younger patients ( $<40$ years) suffering from SSI is much higher compared with older patients (71-80 years), 309,636\$ versus $32,205 \$$ respectively.

This study showed for the first time that the total health costs, direct costs, for patient of all ages are only $1,084,639 \$$, whether the

*Corresponding author: Dohmen PM, M.D Ph.D, Department of Cardiac Surgery, Heart Center Leipzig, University of Leipzig, Struempellstrasse 39, D-04289 Leipzig, Germany, Tel: +49341865 1432; Fax +49 341865 1452; E-mail: pascal.dohmen@yahoo.de

Received November 23, 2012; Accepted November 24, 2012; Published November 26, 2012

Citation: Dohmen PM (2013) Economic Burden of Surgical Site Infections in Cardiac Surgery. J Med Microb Diagn 2: e120. doi:10.4172/2161-0703.1000e120

Copyright: ( 2013 Dohmen PM. This is an open-access article distributed under the terms of the Creative Commons Attribution License, which permits unrestricted use, distribution, and reproduction in any medium, provided the original author and source are credited. 
Citation: Dohmen PM (2013) Economic Burden of Surgical Site Infections in Cardiac Surgery. J Med Microb Diagn 2: e120. doi:10.4172/21610703.1000e120

total years of productive life lost costs, indirect costs, are 8,090,861\$, means eight times as high which has an enormous impact on the economic burden. Furthermore these data show that younger patients with SSI have an underestimated impact on the economic situation of the society. With this knowledge it will be important to estimate new programmes in which younger patients faster rehabilitate to decrease years of productive life lost. In addition, there are intangible costs such as psychological disability, pain suffering, changes in daily activities which were not addressed and should be of interests for future research.

\section{References}

1. Dohmen PM, Konertz W (2007) A review of current strategies to reduce intraoperative bacterial contamination of surgical wounds. GMS Krankenhaushyg Interdiszip 2: Doc38.
2. Sharma M, Berriel-Cass D, Baran J Jr (2004) Sternal surgical-site infection following coronary artery bypass graft: prevalence, microbiology, and complications during a 42-month period. Infect Control Hosp Epidemiol 25: 468-471.

3. Mekontso-Dessap A, Kirsch M, Brun-Buisson C, Loisance D (2001) Poststernotomy mediastinitis due to Staphylococcus aureus: comparison of methicillin-resistant and methicillin-susceptible cases. Clin Infect Dis 32: 877883

4. Dohmen PM (2008) Antibiotic resistance in common pathogens reinforces the need to minimise surgical site infections. J Hosp Infect 70: 15-20.

5. Leaper DJ, van Goor H, Reilly J, Petrosillo N, Geiss HK, et al. (2004) Surgical site infection - a European perspective of incidence and economic burden. Int Wound J 1: 247-273.

6. Alfonso JL, Pereperez SB, Canoves JM, Martinez MM, Martinez IM, et al (2007) Are we really seeing the total costs of surgical site infections? A Spanish study. Wound Repair Regen 15: 474-481. 